\title{
Más allá de la independencia: editoriales anarquistas colombianas como formaciones culturales y medios alternativos radicales*
}

Alejandra Hurtado Tarazona**

Recibido: 27 de septiembre de 2018 - Aprobado: 5 de marzo de 2019

\section{Resumen}

Este artículo es una reflexión teórica en torno a la categoría de independencia editorial, a partir del análisis de tres editoriales anarquistas colombianas: Pie de Monte Editorial, Imprenta Comunera y Gato Negro; y su papel dentro de un contexto en el que impera la concentración del mercado en grandes conglomerados transnacionales. El objetivo principal es ver cómo, dado que la noción de "independencia" está en constante cambio y engloba en sí proyectos heterogéneos, herramientas teóricas tales como el concepto de formaciones culturales de Raymond Williams, y, en este caso particular, los medios alternativos radicales de John Downing y Chris Atton son precisos para pasar de una visión simplista y estática del campo editorial (independiente-comercial) a analizar las prácticas de proyectos específicos más allá de dichas categorías, pues estos conceptos permiten estudiar las prácticas culturales desde su dinamismo e interconexión con otros procesos sociales. A partir de este enfoque analítico, se concluye que los proyectos anarquistas estudiados ofrecen alternativas a las dinámicas capitalistas neoliberales de las editoriales dominantes, a partir de un modelo de autogestión que apunta hacia un quehacer editorial relacionado con la idea de los comunes (commons).

Palabras clave: editoriales anarquistas, edición independiente, formaciones emergentes, medios alternativos radicales.

* Artículo de reflexión. Derivado de un estudio desarrollado en el marco del doctorado en Creación y Teorías de la Cultura y de una ponencia presentada en el congreso de Latin American Studies Association, Barcelona, 2018. Citar como Hurtado, A. (2019). Más allá de la independencia: editoriales anarquistas colombianas como formaciones culturales y medios alternativos radicales. Análisis, 51(95), 365-388. DOI: https://doi.org/10.15332/21459169.4689

** Magíster en Literatura de la Universidad de los Andes (Colombia). Estudiante de doctorado en Creación y Teorías de la Cultura de la Unviersidad de las Américas Puebla (México). Correos electrónicos: alejandra.hurtadota@udlap.mx; alehurtadot@gmail.com. Orcid: https://orcid.org/0000-0002-8979-0824 


\section{Beyond independence: Colombian anarchist publishers as cultural formations and alternative radical media}

Alejandra Hurtado Tarazona

\section{A bstract}

This article is a theoretical reflection on the category of editorial independence, starting from the analysis of three Colombian anarchist publishers (Pie de Monte Editorial, Imprenta Comunera and Gato Negro) and their role within a context in which market concentration in large transnational conglomerates prevails. The main objective is to see how, given that the notion of "independence" is constantly changing and encompasses heterogeneous projects, theoretical tools such as the concept of cultural formations by Raymond Williams, and, in this particular case, the alternative and radical media of John Downing and Chris Atton, are accurate to move from a simplistic and static view of the editorial field (independent-commercial) to analyzing the practices of specific projects beyond these categories, as these concepts allow studying cultural practices from their dynamism and interconnection with others social processes. As from this analytical approach, we concluded that these anarchist projects offer alternatives to the neoliberal capitalist dynamics of the dominant publishing houses, based on a self-management model that points towards an editorial task related to the idea of the commons.

Keywords: anarchist publishers, independent publishing, emerging formations, alternative radical media. 


\section{Au-delà de l'indépendance: les maisons d'édition anarchistes colombiennes comme des formations culturelles et milieux alternatifs radicaux}

Alejandra Hurtado Tarazona

\section{Résumé}

Cet article vise à comprendre la notion d'indépendance éditoriale au travers de trois maisons d'édition anarchistes colombiennes: Pie de Monte Editorial, Imprenta Comunera et Gato Negro. Il s'agit notamment de saisir leur rôle dans un contexte dominé par les grands groupes internationaux. Si bien que la notion même d'indépendance ne cesse de changer et d'englober des projets très différents, quelques outils théoriques tels que la notion de formations culturelles de Raymond Williams, et celle de milieux alternatifs radicaux de John Downing et Chris Atton, se révèlent importants pour passer d'une vision simpliste et étatique du champ éditorial (indépendant-commercial) à une analyse des pratiques et de projets spécifiques au-delà de ces catégories, car ces autres concepts permettent d'expliquer les pratiques culturelles à partir de leur dynamisme et de leur rapports avec d'autres processus sociaux. Cette approche permet de conclure, enfin, que les projets anarchistes offrent des alternatives aux dynamiques capitalistes libérales des maisons d'édition dominantes, à partir notamment d'un modèle d'autogestion qui vise à créer un savoir-faire éditorial fondé sur l'idée des communes (commons).

Mots clés: maisons d'édition anarchistes, édition indépendante, formations émergentes, milieux alternatifs radicaux. 


\section{Introducción}

Alrededor de los años 60 comenzaron a darse cambios paulatinos en la industria editorial norteamericana, relacionados con un fenómeno de concentración de diversas casas editoriales en unas pocas empresas que cada vez adquirían más poder económico y dominio mediático. Poco después, dicho fenómeno se hizo extensivo a países de Europa, especialmente Francia y Gran Bretaña y, en consonancia con los efectos de la globalización, por los años 80 se volvió una constante a nivel mundial, que hasta hoy caracteriza el panorama de la industria editorial ${ }^{1}$. El mercado del libro en América Latina se ha concentrando cada vez más en dos conglomerados: Planeta y Penguin Random House, los cuales han absorbido las casas editoriales independientes de países como Argentina, Chile, Perú, Colombia y México, entre otros, y han llevado a una crisis de la edición local. No obstante, desde inicios del siglo XXI y hasta la actualidad, en estos mismos países se ha dado una efervescencia de la edición independiente que merece especial atención.

En América Latina se ha venido fortaleciendo cada vez más el estudio de la edición local desde puntos de vista históricos y sociológicos, dado que es innegable la importancia que han tenido editoriales de esta región como Era, Porrúa, Joaquín Mortiz, Sudamericana, Losada, Emecé o Norma, por mencionar solo algunos casos representativos del siglo XX, independientemente de que hayan sido o no compradas posteriormente por grandes grupos. Así mismo, son destacables otras editoriales latinoamericanas que, dentro del mencionado marco de globalización editorial, han logrado consolidarse en el campo editorial del siglo XXI, como Sexto Piso y Almadía en México, Beatriz Viterbo y Mansalva en Argentina, Criatura y Trilce en Uruguay, Alquimia y Cuneta Ediciones en Chile, y las editoriales cartoneras que han formado una gran red, por mencionar solo algunos ejemplos².

En Colombia, la historia de la edición ${ }^{3}$ comenzó a mediados del siglo XVIII con la primera imprenta que llevaron los jesuitas a la Nueva Granada (Cobo, 2000), lo cual indica un surgimiento tardío de la actividad editorial frente a países como México, donde la primera imprenta llegó en 1539. A finales del siglo XIX y XX tres empresas marcaron la industria gráfica: Bedout (Medellín, 1889), Carvajal (Cali, 1904) y Voluntad (Bogotá, 1928); a pesar de los pocos lectores con los que

1 Dos autores clave en relación con el proceso de concentración editorial son Jason Epstein (2002) y André Schiffrin (2005), quienes hicieron estudios importantes en cuanto al proceso de concetración en Estados Unidos y Europa. Por su parte, en cuanto a la concentración de la edición en el mercado de Latinoamérica, estudios como los de Fernando Escalante Gonzalbo (2007) y José Luis de Diego (2007) son muy esclarecedores.

2 Al respecto de estudios sobre edición independiente en Latinoamérica, ver Astutti y Contreras (1998), Saferstein y Szpilbarg (2012), Colleu (2008), Astier (2013), Gómez (2014), López Winne y Malumián (2016).

3 La edición como línea de investigación en Colombia está en proceso de consolidación, bajo el liderazgo del Instituto Caro y Cuervo. Fuera de los reportes gubernamentales que proporcionan valiosa información, trabajos como "Historia de la industria editorial colombiana" de Juan Gustavo Cobo Borda (2000), el número 71 de la revista Lingüística y Literatura dedicado a cultura escrita e impresa en Colombia, con editorial de Diana Guzmán Méndez y Paula Andrea Marín (2017), y "Algunas miradas al libro y a la edición en Colombia" de Margarita Valencia (2015), entre otros, constituyen una base importante para las investigaciones que están en desarrollo. 
contaba el país, dado el bajo índice de alfabetización, tres editores trabajaron como piedra angular de la edición local: Jorge Roa, Germán Arciniegas y Arturo Zapata. No es sino hasta 1960, momento en el que hay más acceso a la secundaria y a la universidad, que el público lector comienza a expandirse.

Esta expansión permitió el florecimiento de la actividad editorial, representada por empresas como La Oveja Negra, Tercer Mundo, Editorial Norma, La Carreta, Cerec, Carlos Valencia Editores y El Áncora. Todas estas editoriales se dedicaron en mayor o menor medida a la edición y difusión de la literatura y el pensamiento locales. (Valencia, 2015, párr. 5)

No obstante, con el tiempo los grupos españoles comenzaron a asentarse (Planeta, Santillana, Ediciones B) y, ante el contexto de crisis política y económica de 19984, las editoriales locales se debilitaron y su punto crítico llegó en el 2011 cuando cerró el Grupo Editorial Norma, que tenía 15 filiales en América Latina.

Sin embargo, en el nuevo milenio también aquí ha nacido una gran cantidad de editoriales independientes, algunas ya posicionadas con un catálogo sólido como Babel, Común Presencia, El Áncora, La Carreta, Diente de León, Ícono, y otras más jóvenes, pero no menos activas y propositivas, como La Iguana Ciega, La Silueta, Sudakuir Editorial, Himpar editores, Chiquitico, Milserifas, Mo Ediciones, Ruge, Animal Extinto, Cardumen, La Jaula Publicaciones, Caín Express, entre muchas otras. Martín Gómez, especialista en edición, en su artículo "La independencia en la edición colombiana: ¿una fuente de valor añadido o un simple eslogan?", señala que las editoriales más jóvenes tienen poco definidas sus líneas editoriales y que no publican con la constancia necesaria para posicionarse como marca, y amenazan su viabilidad y sostenibilidad (Gómez, 2014, p. 19). No obstante, aquí veremos cómo algunos de estos proyectos están motivados por principios muy diferentes a los de trabajar por una marca competitiva dentro del mercado, como los casos que aquí estudiaremos: Pie de Monte Editorial, Imprenta Comunera y Gato Negro.

Algunas de las editoriales mencionadas anteriormente cuestionan el calificativo "independiente", dado que este homogeniza una gran cantidad de iniciativas con principios diferentes, y se autodenominan alternativas, autogestivas, autónomas, emergentes o anárquicas, lo cual señala un enfoque particular y unas lógicas específicas de cada una. Pero, ¿a qué nos referimos al hablar de independencia? Son muchos los debates que se han dado alrededor de esta categoría, evidenciando que, en la medida en que las discusiones sigan moviéndose en términos antinómicos de independiente vs. comercial no habrá herramientas para llegar a análisis más profundos y contundentes de esta cuestión y, sobre todo, que esta

4 Entre 1998 y 2001 hubo en Colombia una profunda crisis hipotecaria y financiera, la cual se dio por la excesiva expansión del sistema financiero a principios de los 90 . 
categoría ha sido manipulada de tantas formas, que ha perdido gran parte de su sentido. Muestra de esto es el trabajo desarrollado por Szpilbarg5:

La intención fue caracterizar lo llamado independiente pensándolo en términos de ser una categoría en transformación constante, además de considerar que en cada etapa al término se le han ido agregando sentidos que por acumulación, han generado que la categoría perdiera un valor explicativo de procesos del campo editorial. Consideramos que esto es así además por la ambigüedad intrínseca y constitutiva de la palabra independencia, que aplicada al ámbito de la cultura, la convierte en un concepto en el que los actores vuelcan diferentes contenidos, según la posición y los capitales que ostenten en el campo y la posición que pretendan alcanzar [...]. pretendimos explorar la hipótesis acerca de la relación creciente entre lo "independiente" y las estrategias de mercado, haciendo que la categoría pierda la fuerza que tenía originalmente, surgida de su asociación con proyectos culturales autogestivos apoyados en redes de resistencia política y cultural. (Szpilbarg, 2015, p. 20)

En consonancia con lo anterior, aquí se postula que la categoría "independiente", dada su actual ambigüedad y cantidad de usos, no proporciona las herramientas necesarias para estudiar la edición como un proceso cultural dinámico. Para poder ir más allá de lo descriptivo (señalar si una editorial depende o no financieramente de otro ente, si publica o no autores desconocidos), esta categoría debe ser nutrida por conceptos como, por ejemplo, los de formaciones emergentes de Raymond Williams, postulados en Marxismo y literatura (2000), y los conceptos de medios alternativos y radicales postulados en Medios Radicales (2009) de John D. H. Downing y Alternative Media (1999) de Chris Atton, los cuales serán utilizados aquí para poder analizar nuestro objeto de estudio.

Para responder la pregunta de investigación, a la luz de los autores mencionados, primero haremos un contexto sobre la edición como objeto de estudio; posteriormente caracterizaremos las editoriales a estudiar, para pasar a explicar los postulados de Raymond Williams, quien, además de las formaciones emergentes, presenta conceptos clave como cultura, prácticas sociales, hegemonía e ideología, los cuales son fundamentales para comprender posteriormente la propuesta de Downing y Atton; finalmente analizaremos hasta qué punto la edición anarquista puede estudiarse a la luz de este modelo, y qué implicaciones tiene.

\section{La edición como objeto de estudio}

¿Por qué es importante estudiar la edición como práctica social y proceso configurador de las obras publicadas? Pensar las obras fuera de sus contextos de

5 Esta autora, en su artículo "Independencias en el espacio editorial argentino de los 2000: genealogía de un espejismo conceptual", hace una historización a dicha categoría de 1998 a 2010 y los debates en torno a esta. 
gestión y producción es perder de vista elementos configuradores de la obra, lo cual indica que el proceso editorial es tan importante como la obra terminada que se encuentra en las librerías; es importante estudiar la edición de textos, en tanto no es solo un modo de llegar al producto final (un libro), sino un proceso que enuncia posturas frente el mundo a partir de la gestión. Nos serviremos de dos ejemplos de autores que han desarrollado este mismo argumento.

En primer lugar está Pierre Bourdieu, quien en Las reglas del arte (1995) hace un seguimiento de las condiciones de producción y circulación de obras francesas publicadas y sus dispositivos institucionales, en aras de caracterizar lo que él va a llamar el campo ${ }^{6}$ literario. Bourdieu expone que, en relación con lo literario, las editoriales juegan un papel básico como mercado de bienes simbólicos (en el que el capital de consagración es lo importante), donde hay apuestas a diferentes ciclos de producción, definidos por el enfoque de la empresa. Bourdieu muestra cómo el campo literario, desde sus inicios, ha sido determinado por un operar económico inherente a una industria cultural ${ }^{7}$, en el que intervienen poderes que afectan la obra (Bourdieu, 1995, p. 319). El autor plantea que la obra responde tanto a su relación con un contexto histórico, político, económico, cultural y religioso, como al sistema de producción, comercio y competencia en el que se da, y define la edición también como un campo.

Es necesario aprehender las relaciones objetivas entre los diferentes agentes que contribuyen a la decisión de publicar [...], pero toda la lógica del campo editorial y la creencia literaria que allí se engendra inclinan a olvidar que las interacciones [...], de las cuales cada microcosmos editorial es el lugar, están determinadas por la estructura del campo editorial en su conjunto. (Bourdieu, 2012, p. 224)

Lo anterior evidencia la importancia de los procesos editoriales como configuradores de la obra y la importancia del campo de la edición ${ }^{8}$ como objeto de estudio.

6 El concepto de "campo" es definido como un espacio social relativamente autónomo, que tiene una lógica y organización específica, el cual se constituye a partir de la relación y tensiones internas entre sus agentes. La razón por la que la teoría de los campos de Bourdieu es indicada para el caso editorial, es que su aparato teórico permite estudiar de manera relacional e histórica el ámbito editorial, no como una cadena de procesos para elaborar un producto final, sino como una práctica social de la cual forman parte múltiples mediaciones y actores, cuyo abordaje es fundamental para analizar un fenómeno complejo que va más allá de la particularidad de cada empresa o proyecto editorial. Para hacer un estudio de la circulación de las ideas que llegan a ser publicadas, es necesario estudiar, además de las ideas en sí, sus modos de producción, materialización, de circulación y difusión, su recepción, los circuitos editoriales por los que pasa y las comunidades intelectuales de las que hacen parte, pues son constitutivas de estas ideas.

7 Término propuesto por Theodor Adorno y Max Horkheimer (1999) en The Culture Industry: Selected Essays on Mass Culture. Aquí los autores señalan cómo en la modernidad los productos culturales se convirtieron en el resultado de un trabajo a gran escala, dadas las posibilidades técnicas y tecnológicas, lo cual llevó a la masificación de los libros, por ejemplo, y a la priorización del rendimiento del capital por encima del valor simbólico y cultural de estos. Si bien la lectura sobre las industrias culturales que dan estos autores es completamente crítica, sin tener en cuenta que este mismo desarrollo tecnológico puede abrir la posibilidad a grandes oportunidades y nuevas herramientas (como ahora lo podemos ver más claramente con los nuevos medios), los puntos de los que parte su crítica al capitalismo están aún vigentes y lo podemos ver claramente en el campo editorial.

8 El concepto de campo editorial se desarrolla detenidamente en el capítulo "Una revolución conservadora en la edición", del libro Intelectuales, política y poder (Bourdieu, 2012). 
El segundo ejemplo es el de "The institutional matrix of romance" (1999), de Janice Radway, quien hace un análisis sobre el éxito de las novelas de romance en Estados Unidos. La autora sostiene que abordar un fenómeno literario pensando únicamente en la relación libro-audiencia no es suficiente, pues hay que tener en cuenta el proceso editorial y las relaciones de este con las agencias de publicidad, las distribuidoras, entre otros, lo cual incide directamente en cómo es concebido el libro como producto.

Book buying, then, cannot be reduced to a simple interaction between a book and a reader. It is an event that is affected and at least partially controlled by the material nature of book publishing as a socially organised technology of production and distribution. (Radway, 1999, p. 504)

La autora muestra cómo la venta de estas novelas no se da fortuitamente porque las mujeres de repente tengan el deseo de fantasear con romance, sino porque hay mecanismos concretos que se ponen en acción para que estos interesen y lleguen a un público definido. Es interesante el seguimiento que hace Radway a la figura del editor, el cual, a lo largo del siglo XIX, tenía una relación cercana e individual con el público lector y con las obras que recomendaba. No obstante, poco a poco, y de la mano con avances tecnológicos que permitieron hacer tirajes más grandes a menor costo, la figura del editor se va volviendo más un estratega de ventas, especialmente a partir de los años 70, cuando ocurre también en Estados Unidos la concentración de las editoriales independientes en grandes grupos. Esto puede extrapolarse al campo editorial latinoamericano, donde cada vez el perfil de los editores de grandes editoriales está más inclinado hacia la mercadotecnia, pues el objetivo principal es acaparar amplios sectores de compradores. Estos ejemplos dejan claro el por qué, además de los catálogos, estudiar los procesos editoriales es relevante, lo cual nos permite entrar directamente a nuestros casos de estudio.

\section{Caracterización de editoriales anarquistas}

A continuación, haremos la caracterización de las tres editoriales que aquí nos ocupan. Metodológicamente, estas editoriales fueron elegidas porque son tres de las cuatro identificadas dentro de la "ola anarquista" de edición colombiana, según corrobora un artículo escrito por Andrés J. López (2017) en la revista de divulgación Cartel Urbano (faltó la editorial El Aguijón, cuyos datos no se incluyen por falta de respuesta a la entrevista). Estas editoriales fueron escogidas por compartir la misma línea editorial, el anarquismo, enfoque que permite entrever una relación entre edición, movimientos sociales y activismo que resulta atípico en el ámbito de la edición tradicional. La información que se presentará a continuación en las tablas 1,2 y 3 está basada en la página web de las editoriales, y en las respuestas dadas a una entrevista semiestructurada que se hizo a cada editor, cuyo enfoque eran los aspectos de gestión. 
Tabla 1. Información Imprenta Comunera

\begin{tabular}{|c|c|}
\hline Información solicitada & Imprenta Comunera \\
\hline Lugar y año de creación & 2016. Bucaramanga. \\
\hline Descripción & $\begin{array}{l}\text { Espacio para la difusión y generación de } \\
\text { propuestas e ideas anarquistas. }\end{array}$ \\
\hline $\begin{array}{l}\text { Hacen parte de alguna asociación o grupo } \\
\text { de editoriales }\end{array}$ & $\begin{array}{l}\text { Como proyecto editorial está anclado al } \\
\text { colectivo Lxs Comunes. Participan en } \\
\text { ferias del libro autogestionadas. }\end{array}$ \\
\hline $\begin{array}{l}\text { Dependen de algún ente privado o } \\
\text { público }\end{array}$ & No. \\
\hline Modo de financiación & Recursos propios. \\
\hline $\begin{array}{l}\text { Comercialización y distribución de sus } \\
\text { libros }\end{array}$ & Librerías independientes. \\
\hline Perfil profesional o no profesional idóneo & No hay perfil específico. \\
\hline Organización interna & Horizontal. No hay líderes. \\
\hline $\begin{array}{l}\text { Particularidades en proceso de } \\
\text { producción }\end{array}$ & $\begin{array}{l}\text { Solo se terceriza la impresión. Libros } \\
\text { hechos a mano. }\end{array}$ \\
\hline Políticas de propiedad intelectual & Anti copyright. \\
\hline Enfoque teórico del anarquismo & $\begin{array}{l}\text { Anarquismo libertario y comunismo } \\
\text { libertario. }\end{array}$ \\
\hline Relación movimientos sociales & $\begin{array}{l}\text { Expresiones del movimiento comunista } \\
\text { libertario y anarquista en Bucaramanga, } \\
\text { Cauca y Bogotá. }\end{array}$ \\
\hline Ejemplos títulos & $\begin{array}{l}\text { Cuentos para irrumpir en la moral (varios } \\
\text { autores), Negros presagios: política anarquista } \\
\text { en la época del colapso (Uri Gordon). }\end{array}$ \\
\hline
\end{tabular}

Fuente. Elaboración propia 
Tabla 2. Información Pie de Monte

\begin{tabular}{|c|c|}
\hline Información solicitada & Pie de Monte \\
\hline Lugar y año de creación & 2016. Bogotá. \\
\hline Descripción & $\begin{array}{l}\text { Proyecto editorial cuyo objetivo es } \\
\text { publicar obras inéditas, principalmente } \\
\text { latinoamericanas. Su propósito es } \\
\text { funcionar bajo dinámicas diferentes } \\
\text { de la lógica mercantil y el fetichismo } \\
\text { presentes en el gran mercado editorial, } \\
\text { reivindicando la elaboración manual. }\end{array}$ \\
\hline $\begin{array}{l}\text { Hacen parte de alguna asociación o grupo } \\
\text { de editoriales }\end{array}$ & $\begin{array}{l}\text { Trabajan en el mismo taller con la } \\
\text { Imprenta Comunera. Participan en ferias } \\
\text { del libro autogestionadas. }\end{array}$ \\
\hline Dependen de algún ente privado o público & No. \\
\hline Modo de financiación & Recursos propios. \\
\hline $\begin{array}{l}\text { Comercialización y distribución de sus } \\
\text { libros }\end{array}$ & $\begin{array}{l}\text { Acuerdos con librerías independientes } \\
\text { (La Valija de Fuego, La Madriguera del } \\
\text { Conejo). }\end{array}$ \\
\hline Perfil profesional o no profesional idóneo & No hay perfil específico. \\
\hline Organización interna & Horizontal. No hay líderes. \\
\hline Particularidades en proceso de producción & Libros hechos a mano. \\
\hline Políticas de propiedad intelectual & $\begin{array}{l}\text { Incentivo anti copyright y piratería para } \\
\text { obras reeditadas. Para obras nuevas, } \\
\text { Creative Commons. }\end{array}$ \\
\hline Enfoque teórico del anarquismo & $\begin{array}{l}\text { Anarco comunismo, anarquismo } \\
\text { posizquierda, municipalismo libertario. }\end{array}$ \\
\hline Relación movimientos sociales & $\begin{array}{l}\text { Colectivo Lxs Comunes, organizaciones } \\
\text { campesinas del Cauca (Colombia). }\end{array}$ \\
\hline Ejemplos títulos & $\begin{array}{l}\text { Ecología y pensamiento revolucionario } \\
\text { (Murray Bookchin), La zona temporalmente } \\
\text { autónoma (Hakim Bey), El anarquismo } \\
\text { (Piotr Kropotkin). }\end{array}$ \\
\hline
\end{tabular}

Fuente. Elaboración propia 
Tabla 3. Información Gato Negro Editorial

\begin{tabular}{|c|c|}
\hline Información solicitada & Gato Negro Editorial \\
\hline Lugar y año de creación & 2008. Bogotá. \\
\hline Descripción & $\begin{array}{l}\text { Editorial antiautoritaria especializada en } \\
\text { teoría historia y literatura anarquista y en } \\
\text { el mundo libertario. }\end{array}$ \\
\hline $\begin{array}{l}\text { Hacen parte de alguna asociación o grupo } \\
\text { de editoriales }\end{array}$ & $\begin{array}{l}\text { Participan en ferias del libro } \\
\text { autogestionadas y apoyan otros proyectos } \\
\text { con el mismo enfoque como Pajarera } \\
\text { Libertaria. }\end{array}$ \\
\hline Dependen de algún ente privado o público & No. \\
\hline Modo de financiación & Recursos propios. \\
\hline $\begin{array}{l}\text { Comercialización y distribución de sus } \\
\text { libros }\end{array}$ & $\begin{array}{l}\text { Acuerdos con librerías independientes } \\
\text { (La Valija de Fuego, La Madriguera del } \\
\text { Conejo). }\end{array}$ \\
\hline Perfil profesional o no profesional idóneo & No hay perfil específico. \\
\hline Organización interna & Horizontal. No hay líderes. \\
\hline Particularidades en proceso de producción & Solo se terceriza impresión. \\
\hline Políticas de propiedad intelectual & Acceso abierto, anti copyright. \\
\hline Enfoque teórico del anarquismo & Anarquismo social, autogestión. \\
\hline Relación movimientos sociales & $\begin{array}{l}\text { Feminismo (La Voz de la Mujer), Pro } \\
\text { Palestina (BDS por Palestina). }\end{array}$ \\
\hline Ejemplos títulos & $\begin{array}{l}\text { Antología anarquista, ¿Qué es eso de la } \\
\text { anarquía? (varios autores), Estrategias y } \\
\text { tácticas en la práctica anarquista (Errico } \\
\text { Malatesta). }\end{array}$ \\
\hline
\end{tabular}

Fuente. Elaboración propia 
Como podemos ver, las editoriales comparten las siguientes características: son todas creadas después del $2000^{9}$, lo cual responde a la mencionada efervescencia de la edición independiente del siglo XXI; Imprenta Comunera y Pie de Monte comparten taller de producción, lo cual les permite reducir costos, $\mathrm{y}$, aunque Gato Negro trabaja sin ser parte de un colectivo, las tres participan colaborativamente en ferias de libros independientes ${ }^{10}$; ninguna cuenta con apoyo externo público ni privado, dado que precisamente de esto parte su propuesta de autonomía ideológica que se da gracias a su autonomía económica; los libros son de bajo costo (por ejemplo, el precio promedio de las publicaciones de Imprenta Comunera es de 3 UDS), se distribuyen en encuentros libertarios y gracias a una red de librerías independiente ${ }^{11}$ que, también desde un enfoque muy diferente al comercial, tienen porcentajes accesibles para los editores y hacen acuerdos en aras no solo de impulsar un negocio, sino de fortalecerse como proyecto de resistencia a través del libro; a partir de sus contenidos apoyan ciertos movimientos sociales como el feminismo, el indigenismo, las causas pro palestina, entre otros, lo cual evidencia el compromiso social que tienen como proyecto que se posiciona frente a los discursos dominantes y que concibe la edición más como práctica social que como negocio; los libros son hechos a mano en dos casos, como postura frente a la mercantilización y producción en masa de las grandes empresas, y en los tres casos hay acceso abierto a los contenidos, también como postura de resistencia frente al copyright, y su noción de propiedad privada del conocimiento. Respecto a este último punto, expresa Santiago López de Pie de Monte:

Podríamos partir de la idea repetida hasta el cansancio de que toda propiedad es un robo. Las obras, las ideas son un producto cultural, histórico, para los que son necesarios infinitos procesos, lo menos que podemos hacer es reconocerlo, por tanto permitir el flujo de las ideas, los materiales, los conocimientos. Con Pie de Monte publicamos literatura emergente además de textos anarquistas y operamos, a menudo, de dos maneras distintas. Los materiales anarquistas son compartidos según nuestra interpretación gráfica y bajo la política del anti copyright, incentivamos, para todos, la reproducción por los medios que se consideren necesarios, celebramos la piratería. Lo mismo para los textos nuevos, emergentes, pero definido dentro del marco de los Creative Commons, para proteger de cierta forma los materiales de usos que descalificamos, por ejemplo la apropiación de los textos con fines exclusivamente

9 En el caso de Imprenta Comunera, esta existía desde antes del 2016 bajo el nombre de Editorial Panclasta; después de algunos cambios en el equipo editorial se ancló al colectivo político Lxs Comunes y cambió de nombre. Este colectivo trabaja en torno a la autonomía y las iniciativas libertarias, y ha realizado encuentros como el "Primer encuentro por la autonomía y la libertad México-Colombia", que se llevó a cabo en 2016 en Bogotá.

10 Si bien la feria del libro independiente más grande es la Feria del Libro Independiente y Autogestionado (FLIA, 2012), a lo largo del año se dan otros encuentros en ferias más pequeñas en casas culturales como Casa Kilele, y ferias de otras ciudades de Colombia como la Feria Anarquista de Medellín. La FLIA fue una iniciativa que comenzó en Buenos Aires en el 2006 y se ha ido expandiendo por varias ciudades de Argentina y por otros países de América Latina como Chile, Uruguay, Paraguay, Brasil y México; es autogestionada, organizada colectivamente, sin apoyos públicos ni privados, desde y para todos quienes estén interesados.

11 Entre estas librerías está La Valija de Fuego, La Madriguera del Conejo y San Librario, entre otras. 
económicos por parte de una editorial que replique las dinámicas que imperan, las del fetichismo mercantil. Es decir que el camino está abierto a procesos que quisieran publicar estos materiales y a personas que quieran reproducirlos, porque consideramos que están en su legítimo derecho, pero el uso que se le pueda dar depende también de las personas que lo han producido. (Pie de Monte Editorial, entrevista 9 de mayo de 2017)

Lo anterior es un ejemplo de cómo existe en estos proyectos una consistencia entre contenidos y gestión editorial, una conciencia del quehacer político del editor que no solo enuncia una posición a partir de su catálogo, sino también a través de sus prácticas y determinaciones en cuanto a, por ejemplo, la propiedad intelectual. Esto se ampliará más adelante.

Es importante mencionar que, si bien los editores se refirieron a diferentes autores al hablar de anarquismo (Bakunin, Malatesta, Goldman), acá no nos detendremos en las diferentes vertientes del anarquismo ${ }^{12}$ como doctrina política, sino que enfatizaremos lo que ese enfoque anarquista deriva en el quehacer editorial: la autogestión, la cual se da gracias a que no reciben financiamiento ni tienen vínculos con empresas públicas ni privadas, y el obrar desde un interés común.

Al hacer referencia a aquello común, aludimos a un concepto clave que desde hace pocos años se está desarrollando como vía alternativa ante el sistema capitalista neoliberal: los comunes (commons). La teoría de los comunes se ha desarrollado por autores como Silvia Federici en Revolution at Point Zero (2012), Peter Linebaugh en The Magna Carta Manifesto: Liberties and Commons for All (2009) o por David Bollier y Silke Helfrich en su edición de The Wealth of the Commons (2012). Este concepto no propone otro modelo económico sino que habla de lo común como aquello que puede pensarse desde lo material (recursos naturales, educación, salud) o desde lo inmaterial (relaciones sociales adentro de una comunidad, conocimiento, internet, invención científica y tecnológica), pero también puede pensarse como modelo y modo de configurar los procesos sociales que atraviesan todos los aspectos de la vida; por ejemplo, el libro The Wealth of the Commons incluye 50 ensayos sobre los comunes, en los que se proponen proyectos para monedas alternativas, maquinaria agrícola de código abierto, bosques comunitarios, comunes de coaprendizaje, comunes de teatro, proyectos de mapeado, procomún urbano, comunes digitales, etc. Lo fundamental de los comunes es poder superar las tajantes divisiones sobre las que opera el mundo (público/privado, racional/irracional, individuo/sociedad), y proponer un nuevo modo de relacionarnos, desde un todo orgánico:

12 Con anarquismo aquí entenderemos, desde lo más amplio del término, el rechazo de cualquier figura de autoridad y la lucha contra ella; adhiriéndonos a la definición de la Real Academia de la Lengua Española, será tomada como la doctrina que propugna la supresión del Estado y la eliminación de todo poder que constriña la libertad individual. Esto, dado que no es el objetivo de este trabajo tomar una posición precisa dentro de dicho concepto, sino más bien ver cuál es el enfoque de cada editorial. Es importante sumar a esa definición, el apoyo al self government, la autonomía, la solidaridad, el igualitarismo, la libertad. Esto se opone a un sistema controlado por el poder privado (empresas) y público (el Estado), donde rigen las jerarquías y las normas, la privatización del capital, de las ideas, la especialización del trabajo, la obediencia a poderes reguladores que operan según sus intereses, entre otros. Lo que acá nos interesa es la relación de este self government y autonomía con la noción de commons, desarrollada por autores como Silvia Federici o Massimo de Angelis entre otros que veremos a continuación. 
We are commoners - creative, distinctive individuals inscribed within larger wholes. [...] We are creatures entirely capable of selforganization and cooperation; with a concern for fairness and social justice; and willing to make sacrifices for the larger good and future generations. As the corruption of the market/state duopoly has intensified, our very language for identifying problems and imagining solutions has been compromised [...]. The commons helps us recognize, elicit and strengthen these propensities. It challenges us to transcend the obsolete dualisms and mechanistic mindsets. It asks us to think about the world in more organic, holistic and long-term ways. (Bollier y Helfrich, 2012, p. 23)

La propuesta de estas editoriales anarquistas, si bien no lo hacen explícitamente, van en esa línea, plantear formas de hacer libros desde lo colectivo, la cooperación, la igualdad de posiciones, la colaboración y la noción de un conocimiento que debe fluir y que nos pertenece a todos. Dicho esto, sobre lo cual volveremos posteriormente, pasaremos a relacionar estas propuestas editoriales con los conceptos de alternativo y radical, a partir de las propuestas de Williams, Downing y Atton.

\section{Formaciones emergentes y medios alternativos}

\section{radicales}

Los conceptos de formaciones y emergencia planteados por Williams son clave, en tanto constituyen una base teórica para entender los medios alternativos y radicales de Downing (2009, 44-445) y Atton (2002, pp. 9-156), quienes dialogan con este autor en su teoría. Hay que partir de un punto fundamental, el concepto de cultura, dado que las formaciones son procesos culturales. En Marxismo y literatura, Williams define la cultura como una construcción de significados compartidos y en disputa (shared and contested meaning) que se da en el conjunto de la cultura ordinaria (participativa, de la que todos hacemos parte) y de la "alta cultura" (la especialización, el arts and learning). El hecho de que sea contested es fundamental, dado que si solo fuera compartido no sería un entramado complejo y no tendría cabida la hegemonía ${ }^{13}$ ni la contrahegemonía, conceptos aquí relacionados con el orden dominante capitalista de la edición comercial y sus respectivas resistencias. Williams defiende que la cultura y la economía se sobredeterminan $^{14}$, y no que la economía determina la cultura como lo piensa

13 Aquí con hegemonía, concepto creado por Gramsci en Selections from the Prision Notebook (1999), nos referiremos a la imposición de prácticas y creencias por parte de un grupo o clase social, las cuales se interiorizan y crean sistemas de control que benefician a dicho grupo o clase sobre los demás. En Gramsci la hegemonía está ligada a las clases sociales dado su compromiso con la revolución comunista; no obstante, en Williams hay un sentido más amplio del término que va más allá de la relación de la clase dominante y el proletariado, pues se relaciona con lo cultural.

14 Williams propone que la superestructura está interconectada con la base, es indisoluble y no algo subordinado, pues la ideología hace parte de los procesos materiales y sociales dado que es práctica; en ese sentido, la imaginación y el pensamiento también son prácticas. 
el marxismo ortodoxo ${ }^{15}$, modelo teórico que cae en un esencialismo en el que todo se reduce a lo material, sin tener en cuenta otros aspectos fundamentales que hacen parte de la superestructura (la ideología) ${ }^{16}$.

La teoría de la cultura de Williams relaciona los procesos culturales con una teoría social, histórica y política. El autor caracteriza los procesos culturales a partir de la tradición, las instituciones y las formaciones. Mencionaremos algunos enunciados básicos al respecto, en primer lugar, la tradición está relacionada con la hegemonía que decide cuál es el "pasado significativo" y lo configura a partir de sus propios intereses, lo cual es el principio del concepto de la "tradición selectiva"; en segundo lugar, las instituciones se identifican como aquellos entes hegemónicos formales culturales, políticos y económicos que evidencian gran parte del carácter constitutivo de una cultura; en tercer lugar, las formaciones son:

Aquellos efectivos movimientos y tendencias, en la vida intelectual y artística, que tienen una influencia significativa y en ocasiones decisiva sobre el desarrollo activo de una cultura, los cuales tienen una relación variable y frecuentemente oblicua con las instituciones formales. (Williams, 2000, p. 139)

En este sentido, las editoriales anarquistas son formaciones, en tanto son abiertamente antiinstitucionales y precisamente por lo que aboga su enfoque es porque exista una autorregulación de la misma comunidad, sin necesidad de ese orden hegemónico que regula la tradición y constituye las instituciones. En relación con su influencia en el desarrollo de la cultura, el objetivo de estas editoriales no es constituir una amenaza para los grandes grupos, ni tener el mismo número de lectores, por cuestiones ideológicas; además, dadas sus condiciones materiales, esto sería imposible. No obstante, al unir fuerzas, este tipo de proyectos sí ejercen alternativas y reflexiones en torno a las dinámicas del capitalismo neoliberal. Por ejemplo, los resultados de esta unión y cooperación se pueden ver concretamente en la Feria del Libro Independiente y Autogestionado (ver nota 11), la cual tuvo su primera edición en 2011 en Bogotá, y ahora va por la octava edición. Cada vez ha crecido y se ha fortalecido, permitiendo que haya un mayor acercamiento de este tipo de propuestas a un público más amplio y que efectivamente se creen otro tipo de relaciones a partir del ejercicio de organizar la feria sin apoyo económico de ningún tipo de institución o empresa, a partir de redes de colaboración entre libreros, editores, ilustrados, lectores, de talleres de autopublicación, libros asequibles, entre otros.

Como prácticas que interrelacionan los procesos de tradición, instituciones y formaciones, Williams estudia los conceptos de dominante, residual y emergente.

15 Este autor hace una crítica no tanto a Marx como autor, sino a las interpretaciones que se han hecho de este, como las de Brecht, algunos exponentes de los estudios culturales británicos y en general los estudios basados en un reduccionismo económico.

16 La ideología aquí es entendida como una articulación de ideas, una visión de mundo que, para Gramsci, tiene una fuerza sobre lo material, en tanto produce prácticas. Por ejemplo, los intelectuales orgánicos trabajan en la ideología desde la superestructura y de ese modo pueden intervenir la base (Gramsci, 1999, p. 704). 
En pocas palabras, lo dominante es lo hegemónico; lo residual se define como experiencias que no pueden ser articuladas en términos de la cultura dominante, pero vienen del pasado, aunque son elementos efectivos del presente (por ejemplo, una lengua indígena, pues no hace parte de lo dominante, pero vive en el presente como remanente del pasado); finalmente, lo emergente se define como la continua creación de nuevos significados, prácticas, relaciones y valores que no están cooptados por lo dominante, y por lo tanto tiene el potencial de transformar y reivindicar a partir de este espacio de significación (Williams, 2000, p. 143 $)^{17}$. Respecto a esto, las editoriales que estudiamos son emergentes porque escapan de los órdenes culturales dominantes, y fueron creadas en el siglo XXI a partir de una resignificación de lo editorial y el libro en tanto objeto cultural, para reivindicar otro tipo modo de relación con el mundo, desde la autonomía ${ }^{18}$.

Dentro de lo emergente hay manifestaciones alternativas y oposicionales. Lo alternativo, nuevas formas o adaptaciones de forma que difieren, pero son reconocidas por lo dominante, no necesariamente es oposicional, esto último implica que no puede ser articulado dentro de una narrativa hegemónica; esto se corresponde, en gran medida, con los conceptos de alternativo y radical que veremos a continuación. Las editoriales anarquistas son claramente oposicionales, dado que su concepción del quehacer editorial es totalmente opuesta a la dominante, desde los contenidos que publican como el modo de producir y gestionar los libros; el anarquismo no está de ninguna manera articulado con las narrativas actuales del capitalismo neoliberal y las grandes empresas editoriales que buscan constantemente figuras de autores estrella que se conviertan en grandes vendedores, campañas publicitarias efectivas y distribución de sus libros en grandes superficies.

Dicho esto, abordamos las teorías de John Downing y Chris Atton, dos teóricos que han estudiado medios en los que, a diferencia de los mainstream, prima la importancia de los movimientos sociales y lo comunitario. Downing, en su texto Medios radicales: comunicación rebelde y movimientos sociales ${ }^{19}$, teoriza sobre los medios radicales (los llama también alternativos radicales) y afirma que estos nacen de la cultura popular ${ }^{20}$ y constituyen prácticas de resistencia a la

17 Es importante señalar que estas categorías no son fijas y pueden mutar. Por ejemplo, una práctica residual puede ser incorporada a lo dominante, a partir de reinterpretaciones, proyección o inclusión de los elementos que representen un riesgo para mantener su orden, y más si el residuo hace parte de un importante aspecto del pasado. Lo emergente también puede ser incorporado por lo dominante o volverse residual. Lo importante acá es notar que no son prácticas estáticas sino dinámicas.

18 Vale aclarar que, aunque el anarquismo como doctrina podría considerarse residual, dado que viene del pasado y no está articulado con la cultura dominante, la propuesta que impulsa de la autogestión y los comunes es emergente en tanto actualmente se está elaborando como una nueva propuesta y modo de relación con el mundo frente al capitalismo neoliberal.

19 Para este trabajo se ha utilizado la traducción de la versión de 2001 de Downing, la cual difiere de la de 1984 , en tanto tiene en cuenta diferentes tipos de medios radicales, aunque siempre enfocado en los movimientos sociales.

20 Al hablar de cultura popular Downing rechaza la clásica y tajante división que hace Adoro y Horkheimer en The Cultural Industry (1999) entre cultura de masas (productos de industrias comerciales como la publicidad) y cultura popular (expresión auténtica de la visión de mundo dada en, por ejemplo, la música o el arte popular), y se refiere más bien a la concepción de Jesús Martín Barbero, quien alude a procesos de hibridación entre estas dos esferas. Por esto, afirma que "la cultura popular y la de masas se interpenetran y riegan la una a la otra, del mismo modo la cultura de oposición bebe de las dos primeras y contribuye a ellas" (Downing, 2009, p. 410). 
hegemonía dominante, cuestionando sus posturas ideológicas (léanse estos términos desde lo explicado en la sección dedicada a Williams). La importancia de estos medios radica en que abren espacios a voces que no pertenecen al orden hegemónico, permitiendo que se nutran los abordajes y perspectivas de la vida social, asociados a una comunicación rebelde. En breve, Downing define las características de estos medios del siguiente modo:

Primero, los medios alternativos radicales expanden la gama de información, reflexión e intercambio de los frecuentemente estrechos límites hegemónicos del discurso de los medios mayoritarios. Esto en parte se logra debido a su abundancia. Segundo, frecuentemente tratan de ser más receptivos que los medios mayoritarios a las voces y aspiraciones de los excluidos. Con frecuencia guardan una cercana relación con los movimientos sociales del momento, y por lo tanto y de modo bastante espontáneo, expresan puntos de vista y opiniones que los medios mayoritarios excluyen o ridiculizan. Con bastante frecuencia lideran al abordar asuntos que los medios mayoritarios tardan en notar. Tercero, los medios alternativos radicales no tienen que censurarse a sí mismos para satisfacer los intereses de los magnates de los medios, del poder estatal arraigado o de la autoridad religiosa. Cuarto, su propia organización interna es a menudo mucho más democrática que jerárquica. Por último, algunos de estos medios cumplen con el papel innovador que Raymond Williams atribuye a lo que denominó formaciones (...). Al unir estos elementos cobra total sentido ver los medios radicales como agentes de poder de desarrollo, no como simples instituciones de contrainformación. (Downing, 2009, p. 448)

Además de lo anterior, dentro de su modelo identifica dos tipos de medio radicales: el modelo leninista (el que tomaron los partidos comunistas a lo largo del siglo XX y llegaron al extremo de la censura y el acoso a la oposición), y el que aquí nos concierne, el modelo de autogestión, donde no hay entes privados ni públicos que controlen y estén a cargo del medio, en nuestro caso las editoriales, sino que hay más bien una autorregulación a la que ya habíamos aludido.

Si bien Downing relaciona su trabajo principalmente con medios como la radio o la prensa, esta caracterización aplica también a la edición como un medio cuya herramienta de transmisión es el libro. Los elementos arriba mencionados se corresponden con los proyectos anarquistas, expanden la gama de información, reflexión e intercambio de los límites hegemónicos a partir de los contenidos que publican; son más receptivos a las voces de los excluidos a partir de su trabajo con movimientos sociales; no tienen que censurarse a sí mismos dada la independencia de entes externos; su organización interna es más anárquica que jerárquica (Downing dice "más democrática", tema que será abordado posteriormente), y cumplen, como vimos, con el papel innovador de las formaciones en tanto proponen otro enfoque completamente diferente al establecido del quehacer editorial. 
Algunos de los elementos mencionados también son fundamentales en la teoría de Atton; no obstante, veremos que en su propuesta, si bien tiene en cuenta como parte importante los movimientos sociales y el activismo (que también relaciona con el término de medios radicales a propósito de Downing), hay campo para otro tipo de iniciativas que no necesariamente están tan ligadas a una causa o activismo particular (los medios alternativos). Atton desarrolla un modelo aplicable a nuevas formas culturales del medio artístico y literario, como las pequeñas editoriales; en su modelo se hace explícita la inclusión de este tipo de iniciativas. En diálogo con Downing, Williams y Bourdieu, estudia qué es un medio radical, qué un medio alternativo, y hace énfasis en la importancia no solo de analizar el contenido de dichos medios, sino el proceso social mediante el cual es construido.

Atton hace una distinción entre medios "radicales" y medios "alternativos", aunque las características que propone cobijan los dos conceptos (se refiere a medios alternativos radicales). Los medios radicales los relaciona con un cambio social revolucionario, y a los alternativos les adjudica una aplicación más general, dado que operan desde lógicas diferentes a las hegemónicas, pero no necesariamente son oposicionales, o radicales.

As a blanket term its strength lies in the fact that it can encompass far more than radical, or 'social change publishing' can; it can also include alternative lifestyle magazines, an extremely diverse range of zine publishing and the small presses of poetry and fiction publishers (Atton, 2002, p. 10).

La categoría de medios alternativos es útil como modelo de análisis para otro tipo de editoriales independientes, cuyo enfoque no esté comprometido con unas problemáticas sociales concretas que se opongan al orden establecido, pero en el caso que nos ocupa los proyectos son alternativos radicales, dado su anarquismo.

En su investigación, Atton hace una juiciosa revisión de las características que han sido propuestas a lo largo del tiempo para que un medio pueda considerarse alternativo y radical, a partir de estudios medulares en el tema como Alternatives in Print, Alternative Journalism, Alternative Media de Michael Traber (1985) o Alternative Media de Tim O'Sullivan (2003), entre otros. Atton señala los elementos más importantes y las limitaciones de estos modelos, lo cual tiene en cuenta al construir su propuesta, que se esfuerza por ser lo bastante amplia como para no homogenizar la diversidad de estos medios.

- Los medios alternativos y radicales representan desafíos a la hegemonía, no solo desde un punto de radicalismo político, sino desde el contenido cultural y estético. De este modo, construyen una realidad que se opone a las convenciones y representación de los medios mainstream. 
- Pueden llevar a cambios sociales por sus mecanismos de producción, que están posicionados respecto a los mecanismos de producción dominantes. Los cambios generados pueden ser a nivel local e incluso individual.

- No operan mediante una estructura jerárquica donde prima la experticia y la competencia. De hecho, se apoya la no profesionalización, la posibilidad de que las funciones se compartan, y de que se puedan aprender y practicar nuevas habilidades de manera autónoma, transformando las relaciones sociales.

- Los modos de distribución son diferentes a los de los medios dominantes, pues, más allá de competir por tener un gran alcance que derive en ganancias económicas, están insertos en unas lógicas de trabajo colectivo donde el objetivo no está planteado en términos de competencia sino de reflexividad a partir de la libre información y el construir en colaboración.

- Cuestionan la noción de propiedad intelectual; son anti copyright porque prima la idea dela información libre y fluida. De ahí que apoyen la reproducción continua por medio de, por ejemplo, fotocopiadoras, para que pueda llegar a los lectores muchas veces de manera gratuita, aunque esto pueda implicar operar en la clandestinidad (violar los derechos de autor).

- Las audiencias tienen un papel activo determinante. En este modelo las audiencias (en nuestro caso los lectores) deben ser creadoras de lecturas oposicionales; deben ser audiencias movilizadas que lleven a vinculaciones horizontales y estructuras organizacionales democráticas; en otras palabras, más que un "público" este modelo busca la inclusión activa de la audiencia o lector, dado el gran potencial de participación de estos medios.

- Muchas veces están vinculados a movimientos sociales donde la información para la acción es parte fundamental del activismo.

- Cuentan con independencia de instituciones públicas y privadas, para poder operar como lugares "libres", autónomos, solidarios y reflexivos.

Atton sintetiza lo anterior en los siguientes puntos:

1. Content (politically radical, socially/culturally radical); news values.

2. Form-graphics, visual language; varieties of presentation and binding; aesthetics.

3. Reprographic innovations/adaptations - use of mimeographs, IBM typesetting, offset litho, photocopiers.

4. 'Distributive use'- alternative sites for distribution, clandestine/invisible distribution networks, anti copyright. 
5. Transformed social relations, roles and responsibilities - reader- writers, collective organization, de-professionalization of e.g., journalism, printing, publishing (Atton, 2002, p. 27).

Como vemos, las editoriales anarquistas pueden pensarse a partir de este modelo, el cual está en consonancia con el de Downing. Valdría la pena agregar respecto al punto que se refiere a los lectores, a quienes no hemos aludido, que en este tipo de proyectos no existe un público previamente estudiado desde la mercadotecnia, como ocurre en ocasiones con las grandes empresas, sino que los contenidos están abiertos a todo quien quiera acceder a ellos; por los temas con los que trabajan, muchas veces ese lector está también ligado con los movimientos sociales, el activismo y los principios libertarios que comparten estos proyectos, permitiendo esa lectura oposicional a la que se refiere Atton.

No obstante, queda un aspecto por mencionar, y es el hecho de que Downing y Atton se refieran a que estos medios y sus estructuras son democráticas. Downing afirma que estos medios tienen una estructura de autogobierno democrático, pensando la democracia desde lo planteado por el profesor en ciencias políticas, Crawford MacPherson, para referirse no a un conjunto de normas para el debate y la negociación, sino a unas implicaciones culturales, políticas y económicas en las cuales prospera el poder de desarrollo. “El concepto de poder de desarrollo se puede usar para complementar las nociones de contrahegemonía y esferas públicas alternativas, y fácilmente puede hacer simbiosis con los distintivos de muchos movimientos sociales" (Downing, 2009, p. 449). En pocas palabras, el poder de desarrollo es el ideal para MacPherson, porque consiste en la posibilidad de que toda persona de una sociedad desarrolle sus capacidades sin que obstáculos no naturales se lo impidan, a partir de una sociedad basada en la libertad. Este es el enfoque que Downing le da a la dimensión oposicional de los medios radicales, los cuales operan desde una democracia que no se da en otras esferas de los medios dominantes.

Por su parte, Atton también se une a la idea de que los medios radicales son democráticos, no jerárquicos, accesibles a la gente del común y no a las élites. Al hablar de democracia Atton alude al sociólogo y comunicólogo Denis McQuail y su modelo de democracia participativa en los medios; un modo horizontal de participación e interacción de una comunidad en los medios, para que haya cabida de voces que no hacen parte del orden dominante. También alude a Williams y su noción de comunicación democrática, en donde los orígenes son múltiples, donde hay acceso común a las fuentes y hay recepción activa (Atton, 2009, p. 8). Las nociones de democracia de los dos autores se centran en el poder colectivo de una sociedad libre, y no en el sistema político que da las normativas para elegir líderes; no obstante, el concepto de democracia no escapa a una institucionalidad de la que hacen parte organismos de poder y se basa en estructuras tradicionales de una sociedad actualmente sometida a las dinámicas del capitalismo. Para dar un paso más allá en la emergencia y radicalidad de este tipo de medios, y más en línea con la autonomía del anarquismo, ¿podríamos dar un giro para pasar de pensar en democracia a pensar en los 
comunes mencionados inicialmente? Esta sería una posibilidad que abriría otro panorama y permitiría analizar este tipo de medios desde nuevas categorías. David Bollier, teórico de los comunes, afirma en una entrevista:

Las instituciones son marcadamente incapaces de evidenciar o expresar las necesidades interiores de las personas y sus anhelos, pero creo que los comunes pueden hacerlo y lo hacen (...) no creo que [la propuesta de los comunes] se trate de algo que precise de una autoridad central para diseñarlo, apropiárselo y después construirlo. Creo que se trata de un proceso de desarrollo más bien biológico, o incluso evolutivo, que se verá animado por muchos principios y dinámicas a pequeña escala. (...) Lo que lo mantendrá unido, en gran parte, será cierto tipo de ética y cultura emergentes que se están empezando a hallar la una a la otra. (Bollier, 2016, párr. 10)

De acuerdo con lo anterior, la propuesta de las editoriales anarquistas como formaciones emergentes y medios radicales podría comenzarse a pensar como una iniciativa que se da en consonancia con el principio de autonomía y cooperación de los comunes, dada su forma de operar, donde se intenta superar la división público/privado, sociedad/individuo, propiedad intelectual, entre otros.

\section{Conclusiones}

Hemos analizado tres proyectos editoriales anarquistas colombianos, a la luz del concepto de formaciones emergentes oposicionales de Raymond Williams, y medios radicales de John Downing y Chris Atton. Las conclusiones de dicho análisis son cuatro: 1) la categoría de edición independiente es insuficiente, dado que su ambigüedad y los múltiples usos que se le han dado a lo largo del tiempo no ofrecen unas herramientas de análisis contundentes que permitan abordar la edición como práctica social dinámica; 2) los conceptos de Williams nutren dicha categoría y son más apropiados para el caso de estudio abordado, en la medida en que están enfocados para entender las prácticas culturales desde su dinamismo e interconexión con otros procesos sociales, y son la base para entender el carácter oposicional de los medios radicales que teorizan Downing y Atton; 3) estos dos autores ofrecen herramientas concretas para analizar la autogestión de las editoriales como medios radicales, y son de gran utilidad en tanto su foco es el modo en que estos medios ofrecen nuevas alternativas ante los medios dominantes, en este caso los grandes conglomerados editoriales; 4) proponemos que el modelo de Downing y Atton tiene estrecha relación con la propuesta de los comunes, dado que los medios radicales propenden por la autogestión, por la no jerarquización, el trabajo colectivo, la relación con movimientos sociales y la autonomía frente al Estado y el poder privado.

Es importante seguir construyendo sobre este último punto, para estudiar las implicaciones de pasar de pensar en democracia a pensar en comunes; tal vez este sería un camino para comenzar a trascender las estructuras binarias tradicionales 
y repensar la edición como un ámbito desde el cual se pueden configurar alternativas frente a los sistemas sociales, políticos y económicos actuales.

Finalmente, como aclaración de las dos primeras conclusiones, cabe resaltar la importancia de aproximarse al ámbito de lo independiente de manera exahustiva, para comprender que esta categoría se encuentra en constante transformación a partir de los diferentes usos que se le da, según el proyecto a la que es aplicada. Es decir, si bien hay cierto consenso de pensar lo independiente referido a proyectos que no hacen parte de grandes empresas y que tienen cierto nivel de autonomía en cuanto a las decisiones de qué publicar o no, para poder conocer el campo editorial a profundidad es necesario tener en cuenta que dentro de esta categoría se encuentran proyectos disímiles cuyas prácticas van más allá de esa elemental definición. En este caso Downing y Atton fueron útiles para pensar los casos de estudios como medios radicales, pero de ningún modo podría pensarse que esto es aplicable a todos los proyectos denominados o autondenominados "independientes", por el simple hecho de que hay algunos que, por ejemplo, reciben apoyos de fondos públicos para sus publicaciones, y esto no podría estar más lejos de los lineamientos de las editoriales anarquistas que acá estudiamos. En cambio, los conceptos de Williams sí pueden servir como herramienta de análisis de los actores dentro de esta categoría, porque precisamente alude a los constantes cambios que se dan de los proyectos, cuyas políticas y prácticas pueden ir cambiando a lo largo de su trayectoria. Tener en cuenta lo anterior es de gran importancia, en tanto se encuentra gran variedad de textos aludiendo a la independencia como la antinomia de lo comercial o lo mainstream, pero hacen falta más estudios que indaguen qué está pasando dentro de esto que se suele percibir como un grupo homogeneo en el campo editorial colombiano; cuáles son las prácticas y posicionamientos de quienes allí se encuentran, cómo se diferencian unos de otros y cómo afectan el espacio social del que hacen parte.

\section{Referencias}

Adorno, T. y Horkheimer, M. (1999). The Culture Industry: Selected Essays on Mass Culture. Londres, Nueva York: Routledge.

Astier, P. (2013). Los repertorios de acción de la independencia. La cooperación entre los pequeños editores independientes, condición para la diversidad lingüística. Comunicación y Medios, 27, 158-162. DOI: https://doi. org/10.5354/0716-3991.2013.27261

Astutti, A. y Contreras, S. (1998). Editoriales independientes, pequeñas. Micropolíticas culturales en la literatura argentina actual. Revista Iberoamericana, 67(97), 767-780. DOI: https://doi.org/10.5195/reviberoamer.2001.5856 
Atton, C. (2002). Alternative Media. Thousand Oaks, CA: Sage Publications.

Bollier, D. y Helfrich, S. (eds.). (2012). The Wealth of the Commons, a World Beyond Market and State. Amherst: Strategies Group.

Bollier, D. (2016). Pensar desde los comunes: entrevista a David Bollier. Eldiario. es. Recuperado de www.eldiario.es/interferencias/Pensar-comunesentrevista-David-Bollier 6 489661061.html

Bourdieu, P. (1995). Las reglas del arte. Génesis y estructura del campo literario. Barcelona: Anagrama.

Bourdieu, P. (2012). Intelectuales, política y poder. Buenos Aires: Eudeba.

Cobo, J. G. (2000). Historia de las empresas editoriales de América Latina siglo XX. Bogotá: Cerlalc.

Colleu, G. (2008). La edicion independiente como herramienta protagonica de la bibliodiversidad. Buenos Aires: La Marca Editora.

De Diego, J. L. (2007). Políticas editoriales y políticas de lectura. Anales de la educación común, 3(6), 38-44.

Downing, J. (2009). Medios radicales: comunicación rebelde y movimientos sociales. En J. M. Pereira y A. Cadavid (eds.), Comunicación, desarrollo y cambio social (pp. 393-507). Bogotá: Pontificia Universidad Javeriana.

Epstein, J. (2002). La industria del libro: pasado, presente y futuro de la edición. Barcelona: Anagrama.

Escalante, F. (2007). A la sombra de los libros: lectura, mercado y vida pública. México: El Colegio de México.

FLIA. (2012). ¿Qué es la FLIA? Recuperado de http://feriadellibroindependiente. blogspot.com/p/que-es-la-flia.html

Gato Negro Editorial. (2016). Editorial anarquista colombiana. Recuperado de https://issuu.com/gatonegroeditorial

Gómez, M. (2014). La independencia en la edición colombiana: ¿Una fuente de valor añadido o un simple eslogan?. Boletín cultural y bibliográfico, 86, 15-27. Recuperado de http://www.lecturalab.org/uploads/website/docs/55621-Gomez $\% 20$ Edicion\%20colombiana.pdf

Gramsci, A. (1999). Selections from the Prision Notebook. Londres: The Electric Book Company. 
Guzmán, D. y Marín, P. (2017). Cultura escrita e impresa en Colombia. Lingüística y Literatura, 71, 11-15. DOI: https://doi.org/10.17533/udea.lyl.n71a09

Linebaugh, P. (2009). The Magna Carta Manifesto: Liberties and Commons for All. Berkeley and Los Angeles, California: University of California Press.

López, A. (2017). Editoriales anarquistas que mantienen vivo el espíritu libertario en Colombia. Cartel Urbano. Recuperado de http://cartelurbano.com/ historias/editoriales-anarquistas-que-mantienen-vivo-el-espiritu-libertarioen-colombia

López, H. y Malumián, V. (2016). Independientes ¿de qué?. México: Fondo de Cultura Económica.

Lxs Comunes. (2016). Recuerdos del primer encuentro por la autonomía y la libertad: México-Colombia. Recuperado de https://loscomunescolectivoanarquista. wordpress.com/2016/08/18/recuerdos-del-1er-encuentro-por-la-autonomiay-la-libertad-mexico-colombia-en-la-ciudad-de-bogota/

Radway, J. (1999). The Institutional Matrix of Romance. En S. During (ed.), The Cultural Studies Reader (pp. 564-576). Londres, Nueva York: Routledge.

Saferstein, E. y Szpilbarg, D. (2012, octubre). El espacio editorial independiente: heterogeneidad, posicionamientos y debates: hacia una tipología de las editoriales en el periodo 1998-2010. Primer Coloquio Argentino de Estudios sobre el Libro y la Edición. (Paper). Argentina: Universidad Nacional de la Plata.

Schiffrin, A. (2005). El control de la palabra. México: Era.

Szpilbarg, D. (2015). Independencias en el espacio editorial argentino de los 2000: genealogía de un espejismo conceptual. Estudios de Teoría Literaria, 7, 7-21.

Valencia, M. (2015). La edición en Colombia. Biblioteca Virtual Miguel de Cervantes. Recuperado de http://www.cervantesvirtual.com/portales/ editores editoriales iberoamericanos/edicion en colombia/

Williams, R. (1989). Resources of Hope: Culture, Democracy, Socialism. Londres, Nueva York: Verso.

Williams, R. (2000). Marxismo y literatura. Barcelona: Ediciones Península.

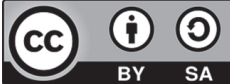

